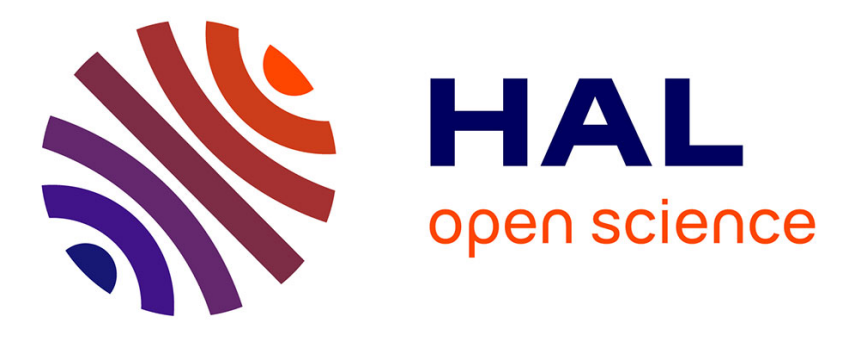

\title{
Le fonctionnalisme linguistique et les enjeux cognitifs
}

\author{
Jacques François
}

\section{To cite this version:}

Jacques François. Le fonctionnalisme linguistique et les enjeux cognitifs. Catherine Fuchs. La linguistique cognitive, Éd. Ophrys; Éd. de la Maison des sciences de l'homme, pp.99-133, 2004, (Cogniprisme), 9782708010352. 10.4000/books.editionsmsh.7063 . hal-00012400

\section{HAL Id: hal-00012400 https: / hal.science/hal-00012400}

Submitted on 21 Oct 2005

HAL is a multi-disciplinary open access archive for the deposit and dissemination of scientific research documents, whether they are published or not. The documents may come from teaching and research institutions in France or abroad, or from public or private research centers.
L'archive ouverte pluridisciplinaire HAL, est destinée au dépôt et à la diffusion de documents scientifiques de niveau recherche, publiés ou non, émanant des établissements d'enseignement et de recherche français ou étrangers, des laboratoires publics ou privés. 


\title{
Le fonctionnalisme linguistique et les enjeux cognitifs
}

\author{
Jacques François
}

Le fonctionnalisme linguistique, fondé initialement sur la notion de marque chez Troubetzkoy (1939), a connu dans les vingt dernières années du $\mathrm{XX}^{\mathrm{e}}$ siècle des développements remarquables dans différentes directions. On peut citer en particulier la lexématique fonctionnelle d'E. Coseriu ( $c f$. Coseriu 2002) ou les théories désignées par W. Croft (1995) comme « fonctionnalistes- formalistes », à savoir la Functional Grammar de S. Dik $(1978,1997)$ et la Grammaire des rôles et de la référence (cf. Van Valin et LaPolla 1997), et avant tout la typologie fonctionnelle des langues développée en majorité par des chercheurs américains, australiens et allemands. Je concentrerai ici mon propos sur des travaux qui mettent en évidence des motivations externes d'ordre cognitif dans la structuration $(\$ 2)$ et l'évolution (§3) des langues et, au-delà, l'ontogénèse (§ 4) et la phylogénèse $(\$ 5)$ du langage. Certains de ces travaux se rattachent explicitement à un programme d'explication fonctionnaliste (en particulier T. Givón, W. Croft, B. Heine ou J. Hawkins, K. Hengeveld, S. Kirby), c'est pourquoi je commencerai par expliciter la controverse entre linguistes formalistes et fonctionnalistes sur le sens qu'ils donnent à la notion d'explication en linguistique (§ 1). D'autres se rattachent indirectement à ce programme comme S. Lamb, R. Van Valin, ou M. Tomasello, mais entrent globalement dans le même paradigme épistémologique.

Je commencerai par une citation emblématique de B. Heine. Dans l'ouvrage de 1997 intitulé The Cognitive Foundations of Grammar, qui synthétise ses travaux sur les processus de grammaticalisation, particulièrement dans un domaine morphologique, celui de l'ap- 
parition des auxiliaires, et dans deux domaines conceptuels, la possession et la comparaison, Heine estime que «l'on ne peut saisir la motivation du mode effectif de structuration du langage que si l'on prend en considération les fondements cognitifs dont dérive la structure du langage » (1997b: 21).

Un an plus tard, dans son introduction au volume collectif The New Psychology of Language consacré aux points de contact entre les orientations fonctionnelles et cognitives de la linguistique contemporaine (rassemblées sous la désignation de « théories du langage fondées sur l'usage »), le psychologue $\mathrm{M}$. Tomasello déclare à son tour que si l'on veut comprendre un jour les complexités multiples du langage, ce n'est pas d'un surplus de formalisations mathématiques que l'on a besoin, mais plutôt de plus de coopération entre psychologues et linguistes pour s'entraider à « déterminer comment les processus cognitifs et sociaux fondamentaux opèrent dans le domaine particulier de la communication linguistique humaine » $(1998:$ XXI).

Nous verrons dans la section 1 en quoi la controverse sur la notion d'explication est directement liée à l'opposition entre une linguistique centrée sur le calcul syntaxique et sur l'hypothèse de l'autonomie de la faculté de langage et une linguistique cherchant à expliquer les structures linguistiques à partir d'un jeu de motivations internes et externes, en l'occurrence cognitives et sociales, lesquelles se combinent harmonieusement dans les thèses de W. Croft ( $c f . \S 3)$ sur l'évolution des langues et de T. Givón $(c f . \S 5)$ sur la phylogénèse du langage.

\section{La notion d'explication dans la controverse entre fonctionnalisme et formalisme en linguistique}

Le fonctionnalisme linguistique contemporain (qu'on peut dire de troisième génération) n'est pas la simple continuation du fonctionnalisme de première génération, représenté par N.S. Troubetzkoy, V. Mathesius ou A. Martinet, consacré en priorité aux structures phonologiques et à l'identification des fonctions du langage (K. Bühler, R. Jakobson), ni de celui de seconde génération orienté 
vers la perspective fonctionnelle de la phrase et l'articulation entre phrase et discours (J. Firbas, P. Sgall, M.A.K. Halliday). Son orientation dominante est typologique, dans la lignée des travaux de J. Greenberg, et celle-ci s'est construite en réaction contre le formalisme d'inspiration chomskienne (et ses présupposés mentalistes). Louvrage programmatique de S. Dik, Functional Grammar (1978), est caractéristique de cette nouvelle voie dont T. Givón est le représentant emblématique. Le fond du débat, c'est la question essentielle du sens donné à l'expression "expliquer un fait linguistique ». C'est pourquoi il serait vain de chercher à caractériser les orientations contemporaines en linguistique fonctionnelle en dehors de cette controverse.

Pour les formalistes d'inspiration chomskienne, un phénomène linguistique est déclaré «expliqué» s'il peut être déduit de l'appariement entre un principe général (formulé dans un vocabulaire de primitives internes à la grammaire) et un ensemble de conditions initiales. Quant aux jugements des locuteurs natifs, ils sont expliqués par l'internalisation de ces principes ( $c f$. Newmeyer 1998 : 97-8). La grammaire générative est donc basée sur un modèle d'explication interne. Le point de vue des formalistes sur le sens dans lequel on peut parler d'explication en linguistique prend appui sur la thèse que les fonctionnalistes confondent abusivement le plan de la compétence, donc de la grammaire interne, et celui de la performance, donc du discours instancié. Les contributions de W. Abraham, S. Anderson et D. Nettle au volume 1 de Formalism and Functionalism in Linguistics (Darnell et al. 1999) sont représentatives de cette position.

\section{1. Aux formalistes la compétence,} aux fonctionnalistes la performance!

C'est dans cette optique qu'Abraham (1999) présente un tableau comparatif d'une douzaine de caractéristiques majeures distinguant les deux entreprises, formaliste et fonctionnaliste. J'en retiens quelques points forts: 
La linguistique cognitive

LES FORMALISTES

appliquent une méthode déductive

pratiquent des généralisations absolues

assignent au langage une fonction primaire d'information

identifient des universaux de syntaxe linguistique

attribuent un caractère arbitraire au lexique

envisagent la diachronie comme constituée de stades successifs

\section{LES FONCTIONNALISTES}

appliquent une méthode inductive

pratiquent des généralisations graduelles ou d'ordre statistique

assignent au langage une fonction primaire d'interaction autant que d'information

identifient des principes cognitifs plus profonds déterminant les universaux linguistiques

attribuent un caractère non arbitraire au lexique, insistant sur l'iconicité, et identifiant de tous côtés des processus métaphoriques

envisagent la diachronie comme un système dynamique

Une démarche calculatoire telle que la préconise le courant formaliste doit adopter une méthode hypothético-déductive, elle ne peut pas se satisfaire de généralisations statistiques, elle ne peut pas tenir compte à la fois des deux fonctions d'information et d'interaction et accorde la priorité à celle qui est le plus aisément formalisable. Elle conçoit l'universalité de la grammaire en termes d'universaux de substance (classes de mots) et non d'universaux implicatifs ou de tendances ordonnées en hiérarchies, elle renvoie la discussion des motivations cognitives du lexique et de l'extension métaphorique des concepts lexicaux comme des catégories grammaticales à une grammaire de performance, enfin elle admet le principe des coupes de synchronie décrivant des états de langue stabilisés comme prérequis de l'analyse diachronique. La prise en compte des motivations pragmatiques de l'énoncé, de ses conditions d'emploi en contexte discursif ou de son appartenance à un genre textuel constituent une tâche secondaire. Abraham y voit « une division raisonnable du travail » (1999: 78).

Dans le même ouvrage, S. Anderson (1999) pousse à son terme cette ligne de raisonnement. Il reproche aux fonctionnalistes de 
ne pas se contenter de formuler l'hypothèse d'une perméabilité des structures syntaxiques vis-à-vis de facteurs de signification et d'usage, mais de concevoir la mise en évidence de cette perméabilité comme une stratégie de recherche (ibid: 120). Selon Anderson, la méthode des formalistes consiste à formuler l'hypothèse que des composantes distinctes du savoir linguistique ont un fonctionnement autonome et que les phénomènes complexes sont descriptibles par leur interaction. L'étude de la capacité générative du langage humain (objectif déclaré des formalistes) invalide les données sur l'usage et la fréquence "et même la question de savoir si une possibilité donnée est effectivement instanciée dans une quelconque langue » (ibid. : 120-121). Le domaine de recherche des formalistes est ce que le langage est (la compétence), tandis que le domaine qu'il concède aux fonctionnalistes est celui de ce que le langage fait (la performance), qu'Andersen caractérise comme la "prise en compte unifiée de l'activité des utilisateurs du langage humain ». Anderson entend fixer une hiérarchie des priorités:

- Les grammaires (internes) de la compétence (ou I-grammars, relevant de modèles formels) sont le noyau dur fondé sur la distinction entre principes et paramètres. Elles sont seules aptes à rendre compte de l'aptitude humaine à manier les langues comme un outil abstrait déconnecté des besoins de communication.

- Les grammaires (externes) de la performance (ou E-grammars, relevant de modèles fonctionnels) rendraient compte de l'usage effectif des langues dans la communication, ce qui selon Anderson constitue une problématique extérieure à la grammaire au sens étroit.

Enfin, la contribution de D. Nettle dans le même volume (1999) prend le problème à la racine en pointant les difficultés du fonctionnalisme en biologie, lesquelles se répercutent sur son emploi en linguistique. Tout comme Givón (1995), dont le fonctionnalisme est pourtant indiscutable, Nettle critique le dogme fonctionnaliste de la structuration adaptative (emprunté à la théorie de l'évolution en biologie). Il présente comme circulaire et empiriquement improductive l'hypothèse de l'adaptativité linguistique - c'est-à-dire d'une relation fonctionnelle entre le degré d'utilité communicationnelle ou cognitive de formes linguistiques et la probabilité de 
leur adoption ${ }^{1}$ et il illustre l'objection de Lass (1980) selon laquelle « le problème avec l'argument fonctionnel, c'est que vous ne pouvez pas perdre » en ces termes: «D'un côté on suppose que le système linguistique est mieux adapté que le précédent après le développement en question et on en trouve une raison a posteriori. D'un autre côté le développement est dit avoir été causé par cette pression fonctionnelle $»$ (Nettle $1999: 450)$.

Comment les fonctionnalistes peuvent-ils répliquer à ces critiques convergentes?

\subsection{Le point de vue fonctionnaliste}

Dans le même volume, le point de vue fonctionnaliste sur ce qui constitue proprement une explication en grammaire est défendu particulièrement par M. Durie (1999) et D. Payne (1999). Durie (ibid.: 418) est largement en accord avec Abraham (cf. § 2.1) sur les bases générales du désaccord avec le structuralisme en général, mais plus spécialement avec l'héritage saussurien. Selon les fonctionnalistes:

- la structure du langage ne peut être expliquée et internalisée qu'en termes de discours et de parole, ce qui contredit le souci des formalistes de se cantonner au niveau de l'unité-phrase et de ne tenir compte que de la fonction d'information au détriment de la fonction d'interaction;

- les contraintes du monde réel, du temps et de l'espace qui s'appliquent à la communication humaine (par exemple l'intentionnalité humaine, et les contraintes imposées par le medium de la communication, verbale

1 Cette critique concerne uniquement les tenants du fonctionnalisme intégratif et, à l'intérieur même du camp fonctionnaliste, Croft exprime les mêmes réticences à l'égard des thèses intégrativistes. II souligne qu'il n'a pas encore été démontré que les motivations concurrentes postulées par les typologistes soient le résultat d'interactions communicationnelles entre locuteurs et qu'aucune expérience psycholinguistique n'a encore prouvé que l'économie et l'iconicité sont bien, comme l'affirme Haiman, les deux moteurs principaux de la structuration linguistique (1995: 515). 
ou signée) doivent être prises en compte ${ }^{2}$, ainsi que les contraintes de l'appareil phonatoire;

- il en est de même des aptitudes et des propensions des êtres humains au-delà du domaine strictement linguistique, y compris les opérations du sens commun et les présuppositions qui rendent la communication coopérative, ce qui va à l'encontre d'une conception restrictive de la compétence limitée à l'usage collectif d'une même syntaxe et d'un lexique arbitraire, abstration faite des extensions métaphoriques affectant aussi bien la syntaxe ${ }^{3}$ que le lexique;

- les systèmes linguistiques sont des équilibres dynamiques et non des états idéalement stabilisés.

Durie développe ce dernier point ( $i b i d .: 426$ ) en distinguant cinq niveaux de temporalité dans le langage, du plus rapide, le discours comme activité individuelle et collective en « temps réel », au plus lent, l'évolution de l'espèce (phylogénie), en passant par la pratique, l'entraînement et la mémoire à long terme, l'acquisition (ontogénie) et l'évolution des langues à travers le renouvellement des communautés linguistiques (diachronie). Je reviendrai sur ces trois types de durée respectivement dans les sections 5, 4 et 3 .

Cet article ouvre la voie à l'argumentation de D. Payne, dont la contribution intitulée What Counts as Explanation? touche le fond du problème. D. Payne commence par définir le fonctionnalisme linguistique comme « une approche qui considère le langage comme un outil de communication et dans lequel la production en cours peut être affectée par les tâches communicationnelles à accomplir vis-à-vis de l'allocutaire aussi bien que par l'appareil cognitif général du locuteur et de l'allocutaire et par les contraintes qui leur sont imposées dans le traitement du langage » (1999: 139). Les trois facteurs décisifs mis en avant par M. Durie sont

2. Ce qui est plus particulièrement développé dans la Cognitive Grammar de R. Langacker et rappelle que celle-ci constitue un embranchement des grammaires fonctionnelles (cf. Croft 1993). Les travaux de B. Heine (1993, 1997a, 1997b; Heine et Kuteva 2002), qui dégagent un catalogue fini de schèmes cognitifs sources de grammaticalisation à travers les langues, vont dans le même sens.

3. La notion d'extension métaphorique de l'agentivité a été particulièrement développée par T. Givón (1989). 
présents : l'accomplissement des tâches communicationnelles, l'appareil cognitif des interlocuteurs et les contraintes auxquelles est soumise la communication linguistique, que celles-ci soient d'ordre spatio-temporel ou physiologique.

Le point central est que les tâches communicationnelles assumées par les locuteurs ont le pouvoir de façonner le système linguistique. C'est l'identification de ce pouvoir qui constitue une explication fonctionnelle selon Russell Tomlin (1994), lequel définit comme description fonctionnelle l'analyse détaillée des relations de correspondance entre les formes spécifiques dans un système linguistique et les fonctions qu'encodent de telles formes. Cette description consiste en un effort de compréhension des motivations des locuteurs dans leur choix entre des structures équivalentes en termes de logique propositionnelle, mais formellement distinctes ${ }^{4}$. Tomlin distingue deux types majeurs de relation entre forme et fonction:

- le codage syntaxique ou grammaticalisé: la forme $\mathrm{X}$ est grammaticalisée pour coder la fonction A si et seulement si la présence de A dans le message requiert du locuteur qu'il emploie automatiquement et invariablement $\mathrm{X}$;

- la signalisation pragmatique: la forme $\mathrm{X}$ signale pragmatiquement la fonction $\mathrm{A}$ si la présence de $\mathrm{X}$ permet à l'allocutaire d'inférer A dans un contexte donné; toutefois, il n'y a pas d'obligation automatique pour le locuteur à produire $\mathrm{X}$ dans le contexte de $\mathrm{A}$.

À titre d'exemple de cette distinction, on peut comparer l'expression en français de la focalisation d'un constituant actantiel ou circonstanciel selon que la force illocutoire est déclarative ou interrogative. En phrase interrogative, la focalisation passe obligatoirement par la sélection d'un pronom ou déterminant interrogatif (par exemple: qui as-tu rencontré?/qui est-ce/c'est que tu as rencontré?/tu as rencontré qui ?/quel $\mathcal{N}$ as-tu/tu as rencontré?), il s'agit donc d'un codage grammaticalisé. En phrase déclarative, la focalisation peut passer par une construction présentative, mais il ne s'agit que d'une signalisation pragmatique: C'est Paul que j'ai rencontré.

4. À ce titre, les règles de "linking " entre syntaxe et sémantique de R. Jackendoff (2002) ont aussi un caractère "fonctionnel". 
Ce type d'explication a pour Newmeyer (1998) un caractère externe - ce qui justifie la distinction de Croft (1995) entre le fonctionnalisme «autonomiste» de S. Kuno, qui ne recourt qu'à des facteurs explicatifs internes au système sémiotique de la langue, et le fonctionnalisme « externe» qui fait intervenir des facteurs pragmatiques, physiologiques et cognitifs.

\subsection{La part d'explication interne et d'explication externe} dans les théories formalistes et fonctionnalistes

Un intérêt majeur de la discussion des modes interne et externe d'explication en linguistique dans le chapitre 3 de Newmeyer (1998 : 96-106) est que, après avoir reconnu que typiquement les formalistes favorisent l'explication interne et les fonctionnalistes l'explication externe, l'auteur montre qu'en fait les deux modes d'explication coexistent dans les deux types de théories.

(I) Explication interne en linguistique fonctionnelle. Ainsi, le principe selon lequel la grammaticalisation est unidirectionnelle, essentiel dans la perspective fonctionnelle, constitue une explication interne parce que c'est une hypothèse interne à la grammaire. Newmeyer note qu'il est habituel de faire des déductions sur des changements dans des grammaires pour lesquelles nous n'avons aucune attestation directe, comme dans l'œuvre de B. Heine où des séquences complexes de développement historique sont proposées pour beaucoup de langues d'Afrique ( $c f$. Heine et Kuteva 2002).

(II) Linnéité comme explication externe en grammaire générative. Quand on essaie, comme les formalistes, d'expliquer pourquoi des grammaires sont organisées comme elles le sont, en faisant l'hypothèse que certaines de leurs propriétés sont innées, on cherche à fournir une explication externe de celles-ci. C'est d'ailleurs pourquoi il n'y a pas à s'étonner que M. Durie (1999) mette en avant dans les préoccupations des fonctionnalistes les caractères innés de la faculté de langage et que toute une littérature soit consacrée à l'acquisition phylogénétique de ces caractères innés ( $c f$. Elman et al. 1996). Martin Haspelmath (cité par Newmeyer) imagine d'ailleurs qu'il est réellement plausible 
que des propriétés innées de l'esprit humain obligent les langues à changer de manières particulières.

(III) L'effet de l'analyse sur les structures syntaxiques. Enfin, comme on peut s'y attendre, la performance linguistique n'est pas sans effet sur la compétence. La plupart des grammairiens générativistes pensent que les grammaires mentales admises par les linguistes sont réellement utilisées dans le cours de la production et de la compréhension du discours, certaines propriétés grammaticales pouvant être attribuées à une accommodation de la grammaire à l'analyseur.

En conclusion de cette discussion, entre les deux modes d'explication linguistique, le mode interne est privilégié chez les formalistes parce que mieux adapté à un style d'argumentation déductif et calculatoire, et le mode d'explication externe chez les fonctionnalistes parce qu'intégrant mieux l'activité linguistique dans le comportement global des êtres humains, de la physiologie phonatoire à la cognition. Pour autant, l'un et l'autre mode d'explication, loin de s'exclure, constituent des procédures scientifiques complémentaires.

\section{Facteurs cognitifs dans la conception fonctionnaliste des universaux du langage}

La conception «formaliste » - plus spécifiquement générativiste des universaux du langage est centrée sur la question des conditions d'acquisition des structures linguistiques par l'enfant malgré la « pauvreté du stimulus ». Chomsky (1965 : 27-30) distingue deux types d'universaux linguistiques: les universaux substantiels sont les pierres angulaires de la structure linguistique, il s'agit des traits distinctifs phonologiques et de la notion de syllabe, des parties du discours (qui sont organisées ultérieurement en fonction de deux traits $[+/-\mathrm{N},+/-\mathrm{V}]$, les noms recevant la double caractérisation $[+\mathrm{N},-\mathrm{V}]$, les verbes $[-\mathrm{N},+\mathrm{V}]$, les adjectifs $[+\mathrm{N},+\mathrm{V}]$ et les adpositions $\left.{ }^{\bullet}[-\mathrm{N},-\mathrm{V}]\right)$, et le principe de hiérarchisation arborescente des syntagmes. Le second type est celui des universaux formels qui constituent le répertoire des types de règles que l'enfant doit assimiler. Dans cette 
conception, l'enfant dispose d'un répertoire de règles (par exemple de formation des syntagmes et de dérivation) qui se développeront en règles de la grammaire interne de l'enfant une fois alimentées par la perception des régularités de la langue maternelle. À ces deux types, Jackendoff (2002 : 77-78) adjoint un universel architectural: selon sa théorie de l'architecture parallèle du langage, toute langue présente une architecture composée de trois types de structures, phonologique, syntaxique et sémantique-conceptuelle, interconnectées par des composantes d'interface phonologie-syntaxe et syntaxe-sémantique et présentant chacune des sous-composantes modulaires ( $c f . \S 6)$.

Le trait commun aux points de vue fonctionnalistes est la mise en avant de données de typologie des langues plus ou moins explicitement corrélées à des observations cognitives et à l'hypothèse que l'acquisition du langage se fonde sur des principes généraux du développement cognitif et que la faculté de langage n'est pas soumise à des principes spécifiques. Je n'évoquerai ici que quatre aspects parmi d'autres de la mouvance fonctionnaliste en termes d'universaux: la notion d'universaux implicationnels ${ }^{\bullet}$, la place de la concurrence entre motivations dans l'émergence de hiérarchies implicationnelles universelles, l'exploitation typologique de schèmes cognitifs universels et l'élaboration d'un ensemble de dimensions universelles très abstraites du langage.

\subsection{Les universaux implicationnels d'ordre des mots et les contraintes de traitement}

Lidentification des universaux implicationnels du langage, qui repose sur les travaux fondateurs de J. Greenberg $(1963,1966)$, s'effectue en quatre pas successifs ( $c f$. Croft 1990 : 62-3). Le premier pas est l'énumération des paires de propriétés dont la compatibilité est testée dans un échantillonnage aussi représentatif que possible de langues. Pour une paire de propriétés $\{\mathrm{A}+/-, \mathrm{B}+/-\}$ le croisement des valeurs $\mathrm{A}+/$ et $\mathrm{B}+/$ - est représenté sur une table dite « tétrachorique » (tableau1). 
Tableau 1

La forme des tables tétrachoriques

\begin{tabular}{|l|l|l|}
\hline & Propriété A + & Propriété A- \\
\hline Propriété B + & & \\
\hline Propriété B - & & \\
\hline
\end{tabular}

Le deuxième pas est la découverte de la distribution empirique des types attestés et non attestés, illustrée par la disposition dans la table des $\mathrm{N}$ (nombre de langues de l'échantillon présentant la paire de propriétés) et des Ø (aucune langue attestée) ou [Ø] (nombre insignifiant de langues attestées). Trois possibilités se présentent:

(I) Il s'agit d'un universel absolu, par exemple: « Toutes les langues ont des voyelles orales » (alors qu'elles peuvent avoir ou pas des voyelles nasales) $)^{5}$ :

Tableau 2

Luniversel absolu de la présence de voyelles orales

\begin{tabular}{|l|c|c|}
\hline voyelles & nasales + & nasales - \\
\hline orales + & $\mathrm{N}$ & $\mathrm{N}$ \\
\hline orales - & $\varnothing$ & $\varnothing$ \\
\hline
\end{tabular}

(II) Il s'agit d'un universel implicationnel, par exemple: «Si une langue dispose le nom avant le démonstratif, alors elle dispose le nom [i.e. l'antécédent] avant la proposition relative »:

Tableau 3

L'universel implicationnel N Dem $\rightarrow$ N Rel

\begin{tabular}{|l|c|c|}
\hline & Dem N & N Dem \\
\hline Rel N & N & $\varnothing$ \\
\hline N Rel & N & N \\
\hline
\end{tabular}

5. Si une seule cellule de la table tétrachorique est validée, on est en présence de la combinaison de deux universaux absolus, par exemple: "Toutes les langues ont des consonnes et ont des voyelles. " 
(III) Il s'agit d'une équivalence logique, par exemple: « Dans les langues avec prépositions, le génitif suit presque toujours le nom régissant tandis que dans les langues avec postpositions ${ }^{\circ}$, il le précède presque toujours » :

Tableau 4

Une équivalence logique

\begin{tabular}{|l|c|c|}
\hline & N Gen & Gen N \\
\hline Préposition & N & {$[Ø]$} \\
\hline Postposition & {$[\varnothing]$} & N \\
\hline
\end{tabular}

Le troisième pas consiste à développer une généralisation propre à restreindre la variation dans les types de langues sans l'éliminer (c'est-à-dire qui autorise les différents types de langues attestés en excluant les types non attestés) et à révéler une relation entre des paramètres grammaticaux par ailleurs logiquement indépendants.

Ainsi, concernant les corrélations entre (1) l'ordre relatif du verbe, du sujet et de l'objet pour la proposition, (2) celui du nom, du démonstratif, de l'adjectif et de la relative pour le syntagme nominal et (3) la présence de prépositions ou de postpositions pour le syntagme prépositionnel, il résulte de l'étude typologique d'un échantillon représentatif de 142 langues ( $c f$. Hawkins, 1983 : 26) que sur les 26 langues à ordre dominant $V S O$ (en proposition principale et en mode de phrase déclaratif) de l'échantillon, 24 présentent la configuration $\{V S O$, prépositions, $\mathcal{N}$-Génitif $\}$ dont en outre 19 avec l'ordre $\mathcal{N}$-Adj contre 5 avec l'ordre $A d j-\mathcal{N}$, les 2 langues restantes ayant des configurations excentriques. Pour les 52 langues à ordre dominant SVO, 32 présentent la configuration $\{S V O$, prépositions, $\mathcal{N}$ Adj\}, dont 21 avec en outre $\mathcal{N}$-Génitif contre 11 avec Génitif-N, et 14 la configuration $\{S V O$, prépositions, Adj-N\} dont 8 avec au surplus $\mathcal{N}$-Génitif et 6 avec Génitif-N, les 6 autres langues se disséminant dans des configurations marginales. Enfin, pour les 64 langues à ordre SOV, 52 partagent les trois propriétés $\{S O V$, postpositions, Génitif- $\mathcal{N}\}$ dont 28 avec en outre Adj-N et 24 avec N-Adj, tandis que 11 des 12 restantes partagent les trois propriétés $\{S O V$, prépositions, $\mathcal{N}$-génitif\} avec $\mathrm{N}$-Adj pour 7 d'entre elles et 
Adj-N pour les 4 autres. On peut donc résumer ces données sous la forme suivante où « > » désigne une relation de supériorité quantitative, l'effectif en langues attestées de chaque propriété d'ordre étant indiqué en indice:

$\left\{\mathrm{VSO}_{26}\right.$, prépositions $\left._{25}, \mathrm{~N}-\mathrm{Génitif}_{24}, \mathrm{~N}-\mathrm{Adj}_{19}>\operatorname{Adj}-\mathrm{N}_{7}\right\}$

$\left\{\mathrm{SVO}_{52}\right.$, prépositions $_{33}>$ postpositions $_{19}$, N-Génitif ${ }_{31}>$ Génitif$\left.\mathrm{N}_{21}, \mathrm{~N}-\mathrm{Adj}_{33}>\operatorname{Adj}-\mathrm{N}_{19}\right\}$

$\left\{\mathrm{SOV}_{64}\right.$, postpositions 59 , Génitif- $\mathrm{N}_{53}>$ Génitif- $\mathrm{N}_{11}, \mathrm{~N}-\mathrm{Adj}_{35}$ $>$ Adj- $\left.\mathrm{N}_{29}\right\}$

et on peut en tirer trois conclusions:

(i) Lordre VSO est en corrélation forte avec la présence de prépositions et l'ordre N-Génitif, l'ordre SOV inversement avec la présence de postpositions et l'ordre Génitif-N. Il y a harmonie (Croft 1990 : 55) entre la structure de la proposition et celle du SN, le même ordre tête-modificateur (VSO) ou modificateur-tête (SOV) étant privilégié.

(ii) Lordre SVO est en revanche en corrélation préférentielle mais non décisive avec les prépositions, l'ordre N-Génitif et l'ordre NAdjectif (c'est-à-dire l'ordre tête-modificateur); cette dispersion témoigne de la concurrence entre deux motivations, l'ordre SV étant du type modificateur-tête dans la conception de Tesnière ${ }^{6}$ ou tête-tête dans celle de Chomsky, et l'ordre VO du type tête-modificateur.

(iii) L'ordre N-Adj l'emporte, même pour les langues SOV, c'est-àdire que dans ce cas il y a une dysharmonie entre l'ordre modificateurtête de la proposition (actants ${ }^{\circ}$-verbe) et l'ordre tête-modificateur du $\mathrm{SN}$ (nom régissant-adjectif régi).

Le quatrième pas consiste dans la recherche d'une explication (éventuellement externe) pour les phénomènes de dysharmonie observés. Hawkins propose deux explications, la mobilité (les démonstratifs, numéraux et adjectifs étant plus mobiles que le génitif et la relative) et surtout le poids relatif (heaviness) évalué en nombre de

6. Tesnière (1969: 23-25) distingue deux types purs de linéarisation des constituants, centrifuge (c'est-à-dire dépendant-régissant), et centripète (régissant-dépendant). Les ordres dits centrifuges ou centripètes " accusés " révèlent une corrélation absolue, les ordres dits " mitigés " seulement une corrélation préférentielle entre un ordre dominant pour les syntagmes verbaux et un ordre dominant inverse pour les syntagmes nominaux. 
morphèmes ou de syllabes, avec par ordre de poids croissant le démonstratif et le numéral puis l'adjectif, le génitif et la relative. Dans l'échantillonnage originel plus restreint de Greenberg (1963), sur 11 langues à ordre SOV, deux présentent la relative postposée (ordre dysharmonique) et une la relative à ordre indifférent, et quatre l'ordre N-Adj au lieu de Adj-N attendu. Il est peu vraisemblable que l'ordre N-Adj résulte de la concurrence entre la motivation d'ordre modificateur-tête et celle de poids relatif dans la mesure où l'adjectif régi a peu de chances d'être notablement plus lourd que le nom régissant, il s'agit donc plus vraisemblablement d'adjectifs mobiles dont l'ordre N-Adj n'est que dominant. En revanche, pour les relatives, le facteur de poids relatif est prépondérant et celui de mobilité peu vraisemblable compte tenu de la structure propositionnelle de la relative. Ce facteur de poids relatif est typiquement d'ordre cognitif, puisqu'il met en cause les conditions de traitement, c'est-à-dire l'empan de la mémoire de travail ${ }^{7}$.

\subsection{Les motivations en concurrence} et les hiérarchies implicationnelles universelles: le cas de l'ergativité• partagée

Les langues à cas se distribuent en trois types principaux de marquage casuel. Dans le système nominatif-accusatif, les fonctions syntaxiques de sujet et d'objet déterminent l'assignation du cas : les sujets sont exprimés au cas nominatif, les objets au cas accusatif. Les fonctions syntaxiques suffisent à déterminer le cas. Dans le

7. À titre d'illustration, en allemand un contenu propositionnel peut être rattaché à un antécédent nominal par une construction participiale préposée avec participe final ou par une relative postposée avec pronom initial et verbe conjugé final. On observe que la participiale n'est représentée qu'en langue écrite, car l'attente de l'antécédent constitue une charge excessive de la mémoire de travail à l'oral. En outre, dans de nombreux cas, l'équivalence entre le pronom relatif et la variante courte et tonique du démonstratif favorise le déplacement en seconde position du verbe conjugué dans la relative, ce qui allège également la charge de la mémoire de travail, par exemple: Ich habe einen Freund, der ist mehrmals rund um die Welt gereist (" j'ai un ami, qui/il a fait plusieurs fois le tour du monde "). 
système ergatif-absolutif ${ }^{\bullet}$ la transitivité du prédicat est le facteur déterminant: les sujets de prédicats transitifs sont exprimés par le cas ergatif, ceux de prédicats intransitifs et les compléments d'objet de prédicats transitifs sont exprimés au cas absolutif. Enfin dans le système actifinactif, qui touche un nombre de langues très limité, ce sont les rôles sémantiques qui déterminent l'assignation de marques casuelles: les constituants agentifs" sont au cas « actif », les constituants non-agentifs au cas « inactif».

La variation de marquage du sujet dans le système ergatif en fonction de la présence ou de l'absence d'un objet révèle la concurrence entre deux motivations: le sujet de la proposition à un seul actant peut certes subir l'événement, mais s'il le contrôle, il reçoit un marquage neutre (dit " absolutif », souvent non marqué) qui laissera la place à un marquage ergatif si le patient est mentionné. C'est un facteur d'instabilité qui favorise l'interférence avec des propriétés cognitives de deux ordres, représentationnelles et énonciatives, qui entraînent le phénomène dit d'ergativité partagée. Les premières concernent les classes de représentation des événements (par exemple l'aspect intrinsèque ou " aktionsart" », les propriétés d'actance) et des participants (par exemple le caractère comptable $v s$. massif, animé $v s$. non animé, etc.), les secondes la deixis` temporelle ${ }^{\bullet}$ la visée aspectuelle, les modalités logiques et énonciatives, la référence et la deixis nominales ${ }^{\bullet}$.

La hiérarchie d'animation de Silverstein (1976), qui peut jouer un rôle déterminant dans le marquage casuel hybride, combine effectivement des propriétés cognitives des deux ordres. Le parangon de l'animé est l'allocutaire, suivi du locuteur et d'un pronom de $3^{\mathrm{e}}$ personne. C'est donc la propriété de participation à l'acte de communication qui domine. Le quatrième degré de l'échelle est représenté par les noms propres, lesquels assurent une fonction référentielle (toujours une propriété énonciative), et les degrés inférieurs distinguent les humains, les animaux et les objets inanimés (propriétés représentationnelles). L'ergativité partagée est la combinaison des marquages casuels ergatif et accusatif dans une langue, distribuée à travers ses sous-systèmes. Les langues ERG/ACC présentent donc un marquage casuel hybride. Dans ces langues, la probabilité d'un 
marquage ERG pour l'agent ou, inversement, d'un marquage ACC pour le patient est inversement proportionnelle à leur "naturalité", c'est-à-dire à la conformité entre le statut intrinsèque du participant et le rôle sémantique qu'il doit assumer dans l'énoncé.

Les agents les plus naturels sont l'allocutaire et le locuteur parce qu'ils sont des humains engagés dans l'acte de co-énonciation. Inversement, les objets inanimés sont des patients naturels. Si cette corrélation prototypique entre la classe conceptuelle et/ou énonciative des participants et leur rôle sémantique est respectée, aucun marquage spécial n'est nécessaire. En revanche, plus l'agent ou le patient est éloigné du prototype correspondant, plus il a de chances d'être marqué respectivement comme ERG ou ACC ( $c f$. tableau 5).

Tableau 5

Modèle du marquage casuel hybride accusatif ou ergatif en fonction de la hiérarchie d'animation

\begin{tabular}{|c|c|c|c|c|}
\hline $\begin{array}{c}\text { Hiérarchie } \\
\text { d'animation }\end{array}$ & $\begin{array}{c}\text { degré } \\
\text { de naturalité } \\
\text { de l'agent } \\
\text { de l'énoncé }\end{array}$ & $\begin{array}{c}\text { marquage } \\
\text { casuel de } \\
\text { de l'agent }\end{array}$ & $\begin{array}{c}\text { degré de } \\
\text { naturalité } \\
\text { du patient } \\
\text { de l'énoncé }\end{array}$ & $\begin{array}{c}\text { marquage } \\
\text { casuel } \\
\text { du patient }\end{array}$ \\
\hline $2^{\mathrm{e}}$ personne & +++ & $\varnothing$ & --- & ACC \\
\hline $1^{\mathrm{e}}$ personne & ++ & $\varnothing$ & -- & ACC \\
\hline $3^{\mathrm{e}}$ personne & + & $\varnothing$ & - & ACC \\
\hline Nom propre & + - & $\varnothing /$ ERG & $+/-$ & ACC/Ø \\
\hline Humain & - & ERG & + & $\varnothing$ \\
\hline Animal & -- & ERG & ++ & $\varnothing$ \\
\hline Inanimé & --- & ERG & +++ & $\varnothing$ \\
\hline
\end{tabular}

L'échelle de naturalité de l'agent de l'énoncé, qui va de +++ pour le pronom allocutaire à - - - pour la désignation d'un inanimé, est l'inverse de celle du patient. Un patient naturel investi d'un rôle agentif (et donc causatif ${ }^{\circ}$ ) dans l'énoncé doit recevoir une marque d'ergatif pour compenser sa faible agentivité. Inversement, un agent naturel investi d'un rôle de patient doit recevoir une marque d'accusatif pour neutraliser son agentivité naturelle. Dans les langues à marquage casuel ergatif sur les noms, le marquage ergatif manque fréquemment 
pour la $1^{\mathrm{e}}$ et $2^{\mathrm{e}}$ personne et parfois pour la $3^{\mathrm{e}}$. Blake $(1994: 123)$ mentionne une langue papou, le fore, pour un usage du cas ergatif déterminé par une variante de la hiérarchie d'animation: pronom $>$ nom propre $>$ terme de parenté $>$ humain $>$ animé $>$ non animé.

Toutefois les spécifications représentationnelles et/ou énonciatives des SN ne sont pas la seule cause de partage de l'ergativité. H. Seiler retient deux autres types de paramètres: la différenciation lexicale des verbes («partage verbal») et l'incidence du temps, de l'aspect et du mode du verbe (« partage TAM»). Dans de nombreuses langues, il y a un partage dans le système de marquage casuel en fonction du temps et de l'aspect. Ainsi le géorgien et le laz (langue kartvélienne) ont un marquage actif au passé et nominatif-accusatif au présent. Dans plusieurs langues indo-aryennes (hindi-ourdou, marathi, punjabi) et dans les langues iraniennes pashtou et kurde, le marquage n'est ergatif qu'au parfait ( $c f$. Blake 1994 : 127-129).

Le répertoire des paramètres susceptibles d'affecter un marquage casuel hybride qu'a établi T. Givón (1984 : 153-165) combine des caractères d'ordre cognitif et d'ordre énonciatif: d'un côté le degré de contrôle ou d'intentionnalité de l'agent, le degré d'affectation du patient et le degré de perfectivité de l'événement concernent la représentation mentale des caractères intrinsèques du procès, de l'autre le degré de manifestation du patient (mentionné ou pas), d'achèvement de l'événement (la visée aspectuelle sécante, globale ou accomplie) et le degré de référentialité" et de topicalité• des participants (leur statut de protagoniste ou de figurant) touchent la mise en scène du procès dans l'acte d'énonciation. La présence indissociable de caractères de ces deux ordres était déjà apparue dans la hiérarchie d'animation de Silverstein et se retrouve également dans les facteurs contribuant à un marquage de la transitivité ( $c f$. Hopper et Thompson 1980) et suggère que ces deux ordres de représentation ne sont que deux facettes qui entrent en composition: la représentation des propriétés invariantes d'un type de procès et des types de participants qu'il convoque, et celle des propriétés d'un procès particulier une fois «mis en scène ».

Le repérage des facteurs représentationnels et procéduraux qui structurent les hiérarchies implicationnelles, telles que la hiérarchie 
d'animation de Silverstein ou celle de transitivité de Hopper et Thompson, permet-il d'expliquer ces hiérarchies? C'est ce que S. Kirby (1998) a cherché à montrer à l'aide d'une simulation par réseau connexionniste à propos de la hiérarchie de rattachement des propositions relatives à leur antécédent. Dans toutes les langues du monde, il semble exister un procédé pour relier une proposition dépendante à un syntagme nominal par l'intermédiaire d'un pronom relatif sujet (la fille qui t'a dit cela) ou d'une construction apparentée (ex. la fille vendant des primeurs au marché), c'est l'universel de formation de la relative sujet. Mais certaines langues ne permettent pas la relativisation à partir de l'objet direct (la fille que tu as vue/photographiée sur le magazine) et encore moins la relativisation à partir d'un objet indirect ou d'un circonstant (la fille à qui tu as parlé/avec qui tu sors). On remarque d'ailleurs qu'en français la construction participiale n'est plus possible dans ce cas. Kirby défend l'idée que la hiérarchie d'accessibilité à la formation des propositions relatives est explicable à partir du jeu de deux pressions fonctionnelles concurrentes: la première vise à réduire la complexité structurale (c'est-à-dire la distance entre la position du pronom relatif et celle de la trace de la fonction qu'il assume dans la relative: très courte pour le pronom relatif sujet, très longue par ex. dans fe connais la fille avec le copain de la soeur de laquelle tu veux monter une boîte) et la seconde à réduire la complexité morphologique. La simulation de Kirby montre que, en tenant compte des deux types de motivation en concurrence et en laissant évoluer le système linguistique ainsi modélisé sur un grand nombre de générations de locuteurs, on aboutit à une représentation de l'évolution d'une langue qui peut fluctuer entre un rattachement de la relative à l'antécédent par la seule fonction sujet et un rattachement diversifié par différentes fonctions syntaxiques.

Larrière-plan théorique de la simulation de Kirby est explicitement le processus de la «main invisible $»^{\bullet}($ Keller 1994) qui décrit les conséquences globales du comportement des usagers d'une langue. Les universaux typologiques implicationnels sont " des résultats non intentionnels d'actions humaines » et Kirby interprète les pressions fonctionnelles qui influencent la sélection de variantes linguistiques en concurrence en termes de « lois de 
transformation » entretenant " une analogie intime avec la pensée biologique ». La notion centrale d' "état de langage intériorisé », c'est-à-dire de grammaire individuelle en tant que système cognitif, correspond exactement à la conception de la grammaire défendue par Croft (2000) dans le cadre d'une théorie du changement linguistique par différenciation dans les réplications ${ }^{\bullet}$ d'énoncés et stabilisation sous l'effet de pressions sociales ( $f f . \S 3$ ci-dessous).

\subsection{Schèmes cognitifs universels et variété des moyens d'expression: I'exemple de la relation de possession}

B. Heine, africaniste de formation et l'un des promoteurs principaux de la théorie de la grammaticalisation, défend dans ses ouvrages sur l'auxiliation (1993), sur la possession (1997a) et sur les fondements cognitifs de la grammaire (1997b, Heine et Kuteva; 2002) l'idée que les intuitions modélisatrices de la Cognitive Grammar de R. Langacker ( $c f$. Victorri, ce volume) sont opératoires et méritent d'être testées extensivement. Elles constituent les bases d'un raisonnement explicatif, mais elles ne sont probantes que combinées à un raisonnement typologique. Dans son étude des fondements cognitifs de l'expression diversifiée de la possession, Heine part de quatre hypothèses:

(I) La structure de catégories grammaticales est prédictible dans une large mesure une fois qu'on connaît l'éventail des structures cognitives à partir desquelles elles sont dérivées.

(II) Les catégories grammaticales peuvent être traquées jusqu'aux concepts source sémantiquement concrets.

(III) Pour toute catégorie grammaticale, il n'y a qu'un vivier limité de concepts source.

(IV) Alors que le choix des sources est déterminé primairement par des modes de conceptualisation universels, il est également influencé par d'autres facteurs, spécialement des forces aréales.

Il commence par établir la liste de tous les schèmes cognitifs à la source de la représentation linguistique de la possession prédicative ou attributive: 
(1) schème d'action: $\mathrm{X}$ prend $\mathrm{Y}$

(2) schème de localisation: $\mathrm{Y}$ est à la place de $\mathrm{X}$

(3) schème d'accompagnement: $\mathrm{X}$ est avec $\mathrm{Y}$ (la chambre est avec salle de bain > la chambre a une salle de bain)

(4) schème d'existence: $\mathrm{Y}$ existe par rapport à $\mathrm{X}$ - schème génitif: $L e \mathrm{Y}$ de $\mathrm{X}$ existe ${ }^{8}$ - schème du destinataire: $\mathrm{Y}$ existe pour $\mathrm{X}^{9}$

(5) schème du topique: En ce qui concerne $\mathrm{X}, \mathrm{Y}($ de $\mathrm{X})$ existe

(6) schème de l'origine: $\mathrm{Y}$ existe à partir de $\mathrm{X}$

(7) schème d'équation: $\mathrm{Y}$ est (propriété) de $\mathrm{X}$.

Puis il montre comment la grammaticalisation « décatégorialise » les unités significatives et comment les schèmes sources tendent à se spécialiser dans l'expression d'un type de possession. Ainsi, la construction possessive anglaise « $\mathrm{X}$ has $\mathrm{Y}$ » a une syntaxe transitive parce que cette construction est dérivée du schème d'action et a retenu les principales propriétés associées à ce schème, mais elle ne peut pas se présenter à la voix passive (contrairement à « $\mathrm{X}$ owns $\mathrm{Y}$ »). Cette contrainte révèle un phénomène de « décatégorialisation » (un représentant typique de la catégorie «verbe d'action » doit être compatible avec un mode de présentation active ou passive de l'action, ce qui n'est plus le cas pour have), lequel constitue dans ce cas un degré intermédiaire du processus de grammaticalisation conduisant à l'emploi de have comme auxiliaire modal (X has to $\mathrm{SV}_{\text {Infinitif) }}$ ) ou temporel (X has $\mathrm{SV}_{\text {Participe passé) }}$. La majorité des schèmes employés pour la grammaticalisation de la possession prédicative implique des prédicats dont la signification originelle concerne la localisation, l'existence, etc., ce qui explique pourquoi des moyens d'expression de la possession prédicative ressemblent fréquemment à des moyens d'expression de ces concepts.

Les schèmes sources primaires ont une répartition variable par aire géographique. Dans les langues européennes, ce sont les schèmes 1 d'action et 2 de localisation; dans les langues asiatiques,

8. Par exemple en turc pour " j'ai un livre »: Kitab $<$ livre $>-i m<$ moi $>$ var $<$ existant $>$ 9. Par exemple en breton pour « j'ai un vélo bleu » ur $<$ un $>$ velo c'hlas $<$ bleu $>a m$ $<$ pour moi $>$ eus $<$ est $>$. 
le schème 4 d'existence, plus spécialement sa variante 5 génitive, le schème 6 du destinataire et le schème 7 du topique; et dans les langues d'Afrique le schème 2 de localisation, le schème 3 d'accompagnement et, secondairement, le schème $1 \mathrm{~d}$ 'action. La situation rencontrée dans les langues européennes présente un caractère typologiquement « exotique ». La structure linguistique des constructions du type avoir $(\mathrm{X}, \mathrm{Y})$ rencontrée dans les langues romanes et germaniques (mais pas nécessairement dans les langues slaves, celtes ou finno-ougriennes) est déterminée primairement par les effets du schème d'action (le possesseur est typiquement encodé comme le sujet de la phrase et le possédé comme l'objet), alors que dans l'ensemble des langues du monde ce schème est relativement peu employé comme structure source.

\section{Le poids des motivations cognitives et celui des pressions sociales dans le changement linguistique}

Dans la typologie fonctionnelle des langues récente, l'arrière-plan historique des types de langues se révèle une préoccupation majeure de nombreux chercheurs, au-delà de l'aire limitée de la grammaticalisation illustrée au § 2.3 par l'œuvre de B. Heine. Dans cette perspective, je pense que l'ouvrage récent de W. Croft, Explaining Language Change, sous-titré An Evolutionary Approach (2000) est destiné à soulever l'intérêt de la communauté des fonctionnalistes.

Croft vise une théorie évolutionniste de la sélection des « linguèmes ${ }^{\bullet}$ ", définis comme " unités de structure linguistique concrétisées dans des énoncés particuliers qui peuvent être hérités par réplication» (2000 : 239). Il rejette fondamentalement la vue « essentialiste » des langues caractéristique du structuralisme américain. En biologie, les espèces ne sont plus identifiées au moyen de propriétés structurales essentielles et sont maintenant conçues comme des populations ouvertes à des variations individuelles. L'espèce est ainsi redéfinie comme " une population d'individus se reproduisant par croisement (interbreeding) ». 
Selon Croft, une langue se définit sur la base de l'interaction communicationnelle effective et de la conviction des locuteurs qu'ils communiquent "dans la même langue ». Cette conviction est la source de sa distinction entre les langues « de mêmes parents »sibling languages, dialectes étroitement apparentés que leurs locuteurs tiennent pour des langues différentes - et les langues « polytypiques »- langues vaguement apparentées que leurs locuteurs respectifs considèrent comme une seule langue.

Les trois concepts principaux de la linguistique « évolutionniste » de Croft, l'énoncé, la langue et la grammaire, introduits en relation avec la « théorie généralisée de la sélection » de David Hull (1988), sont comparés à leurs équivalents en biologie évolutionniste.

Un énoncé est « une occurrence particulière, effective, produite par un comportement humain en interaction de communication (c'està-dire une chaîne de sons), telle qu'elle est prononcée, structurée grammaticalement et interprétée sémantiquement et pragmatiquement dans son contexte» (Croft 2000 : 26). Un énoncé est une chaîne structurée de linguèmes. Ceux-ci sont l'équivalent linguistique des gènes en biologie et les linguèmes aussi bien que les gènes sont des instanciations paradigmatiques du concept de réplicateur dans la généralisation de Hull. Ainsi les énoncés instancient des ensembles structurés de réplicateurs tout comme les chaînes d'ADN en biologie.

Une langue est «la population des énoncés dans une communauté de discours » (ibid.). Elle est vue comme un vivier de linguèmes comparable à un vivier de gènes. Les variantes de langue sont des réplicateurs alternatifs et à ce titre équivalents aux allèles en biologie.

Une grammaire est « la structure cognitive dans l'esprit d'un locuteur » qui contient sa connaissance de sa langue et c'est « la structure qui est utilisée dans la production et la compréhension des énoncés » (ibid.). C'est l'instanciation paradigmatique dans la communication linguistique de l'interacteur, c'est-à-dire l'entité qui interagit avec son environnement « de manière à produire une réplication différenciée » (ibid.: 238) et, de ce fait, une sorte d'organisme du langage.

Le locuteur en interaction réplique sa grammaire interne quand il produit des énoncés. Dans un premier temps, des processus cognitifs perturbateurs tels que la réanalyse du message reçu ou des 
interférences entre codes dans des situations de bilinguisme peuvent entraîner des variations dans la réplication. Dans un second temps, la réplication différenciée conduit à l'établissement de variantes divergeant aux niveaux phonologique, lexical et/ou syntaxique, avant que des facteurs sociaux ne pilotent la sélection et la propagation de l'une des variantes du vivier ainsi constitué. Le propos de Croft est donc double:

- en premier lieu il entend mettre le doigt sur l'avantage pour la linguistique fonctionnelle à élaborer un modèle explicatif intégré dans une théorie générale de la sélection adaptative;

- ensuite il suggère que deux méthodes qui considèrent les langues comme des populations plutôt que comme des essences, en l'occurrence la typologie fonctionnelle des langues et la sociolinguistique, peuvent en fusionnant expliquer les deux stades du changement linguistique, la différenciation de variantes résultant des réplications individuelles, puis la sélection d'une variante par le corps social.

\section{L'ontogénèse: comment l'acquisition des relations sémantiques précède celle des relations syntaxiques}

Les thèses d'inspiration fonctionnaliste sur l'acquisition et la transmission ( $c f$. Deacon au § 5.2) du savoir linguistique se présentent essentiellement comme des répliques à la théorie de « l'instinct du langage » de S. Pinker (1994). Dans l'épilogue de Van Valin et LaPolla (1997 : 640-649) intitulé The Goals of Linguistic Theory Revisited, les auteurs critiquent l'idée que « le capital cognitif qui permet aux enfants d'apprendre une langue est autonome ». Leur propos est fondamentalement d'adapter au cadre de la Grammaire des rôles et de la référence la thèse de M. Braine (1992) sur le type de structure innée nécessaire pour accéder à la syntaxe. Braine se propose de discuter la théorie des catégories syntaxiques innées de Pinker qui se présente sous la forme de trois primitives développementales:

(1) un mécanisme d'apprentissage qui recourt à un principe d'analyse de nouveaux matériaux à l'aide de règles anciennes; 
(2) des catégories sémantiques telles qu'argument et prédicat, ainsi que des catégories ontologiques, par exemple objet, lieu, action, événement, etc.;

(3a) des catégories syntaxiques naturelles $\mathrm{N}, \mathrm{SN}, \mathrm{V}, \mathrm{SV}$, etc.

La contre-proposition de Braine consiste à substituer la primitive (3b) à (3a) :

(3b) une tendance à classer les mots et les syntagmes que (1) ne permet pas de classer, comme référant à des instances des catégories de (2).

Ce qu'il rejette, c'est l'idée que « les catégories syntaxiques de langues existent dès le début de l'acquisition du langage comme des catégories innées ayant une réalité biologique et psychologique distincte de leurs membres canoniques » (p. 91). Selon ses observations, les catégories syntaxiques émergent sous l'effet conjoint des primitives (1) et (3b). Ce faisant, il pose une question cruciale à propos de la fonctionnalité du langage: si les enfants commencent par des catégories sémantiques, pourquoi les langues ont-elles des catégories syntaxiques dont l'extension ne correspond pas à celle de ces catégories sémantiques? Il y répond prudemment en listant quelques facteurs critiques de disparité entre catégorisation sémantique et syntaxique:

(I) les limites floues des catégories sémantiques plausibles du langage de la pensée;

(II) le recouvrement et la concurrence entre catégories sémantiques (telles que les rôles sémantiques) et catégories pragmatiques (par exemple les fonctions de topique et de focus) qui doivent être corrélées aux structures syntaxiques;

(III) la sensibilité des apprenants aux similarités phonologiques entre les mots;

(IV) et le nombre restreint de positions structurales distinguables en raison de la linéarité obligée du discours.

À la théorie de Braine dérivant les catégories syntaxiques de catégories sémantiques primaires, Van Valin et LaPolla ajoutent l'observation qu'il y a des patrons interlinguistiques frappants dans l'acquisition des temps et des aspects, des modalités et de la négation. Ainsi les distinctions aspectuelles apparaîtraient toujours avant les distinctions temporelles. Ils interprètent ces observations comme 
un argument développemental pour la stratification adoptée dans la " projection des opérateurs » de leur modèle grammatical, dans laquelle l'aspect est dans la portée de la modalité de base (capacitive, aléthique et déontique ${ }^{\bullet}$, laquelle est à son tour dans la portée du temps et de la modalité épistémique (1997 : 644).

Dans la même veine, Tomasello (2000) décrit une expérience développementale très instructive dans laquelle il a enregistré environ $10 \%$ de tous les énoncés produits par une enfant âgée de deux ans en interaction avec sa mère durant une période de six semaines. Chaque énoncé de la dernière période a été comparé à des énoncés similaires produits par l'enfant antérieurement dans l'enregistrement. Tomasello qualifie les opérations syntaxiques réalisées par l'enfant dans les derniers jours de « fondées sur l'usage » car « l'enfant composait ses énoncés à partir d'un assortiment hétéroclite de différentes sortes d'unités psycholinguistiques préexistantes ». Cela se faisait en coupant et collant les constructions qu'elle mấtrisait auparavant. Les deux plus importantes conclusions de cette expérience sont en premier lieu que les matérieux stockés, récupérés et réutilisés par l'enfant étaient hautement diversifiés, allant des mots simples à des catégories abstraites et à des énoncés ou des patrons syntaxiques partiellement instanciés (p. 77), et que les enfants combinent non seulement les formes linguistiques impliquées mais aussi les fonctions communicatives conventionnelles de ces formes comme le postulent la grammaire cognitive de Langacker ou la grammaire de construction de Fillmore, Kay et Goldberg ( $c f$. Victorri, ce volume).

\section{La prise en compte de contraintes cérébrales dans le traitement et l'émergence du langage}

Le développement récent simultané de différentes techniques de neuro-imagerie fonctionnelle, appliquées entre autres au langage ( $c f$. Houdé et al. 2002, 3e partie, chap. 3-5) et de simulation du comportement linguistique par réseaux neuronaux ( $c f$. Elman et al. 
1996; Kirby 1998) a développé l'intérêt pour les contraintes d'ordre neurolinguistique et permis des avancées dont je n'évoquerai que deux facettes, la modélisation de la variation individuelle dans la mise en relation du sens et de l'expression $(\$ 5.1)$ et celle des phases probables de la phylogénèse du langage $(\$ 5.2)$.

\subsection{Une linguistique " neurocognitive " pour rendre compte de la variation interindividuelle dans le traitement du langage}

S. Lamb est connu pour avoir conçu dans les années 1960 ( $c f$. Lamb 1966) un modèle de grammaire «stratificationnelle » qui, comme le souligne Jackendoff (2002 : 128) était la première esquisse d'une théorie multigénérative. En effet, dans la version la moins sophistiquée, chacune des strates phonémique, morphémique, lexémique et sémémique (Lamb était originellement un adepte de la tagmémique• de K. Pike) présentait une composante combinatoire, respectivement la phonotactique (combinant les phonèmes en morphèmes), morphotactique (combinant ceux-ci en lexèmes), lexotactique (combinant les lexèmes en propositions) et sémotactique (combinant les sèmes lexicaux en sémèmes). Le modèle a connu moins de succès que la théorie SensTexte assez apparentée de I. Mel'chuk, sans doute parce que les représentations multistratiques, évoquant des tableaux de connexions électriques, étaient d'un maniement difficile. Mais, dès 1971, Lamb évoquait la validité cognitive des réseaux de combinaison des unités phonémiques, morphémiques et lexémiques et de la corrélation entre lexèmes et sémèmes. Et, en 1999, il a fourni une version explicitement «neuro-cognitive » de sa théorie en la combinant avec le modèle «néo-associationniste » de traitement neurophysiologique du langage de N. Geschwind (1965).

Lamb (1999 : 140, figure 8-10) distingue d'une part trois sous-systèmes linguistiques, celui de la production grammaticale précédant la production phonologique, celui de la reconnaissance phonologique précédant inversement la reconnaissance grammaticale et 
celui des connexions lexicales abrégé en "lexis », et d'autre part quatre sous-systèmes extralinguistiques, de perception somatique (ou proprioception), de perception auditive et de perception visuelle (sur trois niveaux de repérage en deux dimensions, deux dimensions et demie, et trois dimensions). Les trois sous-systèmes linguistiques sont en rapport avec des catégories conceptuelles d'un premier niveau, dites catégories objectales, et à l'inverse des catégories de production articulatoire en rapport avec les mécanismes de la parole. La lexis sert d'interface entre les catégories objectales et les catégories abstraites qui constituent le second niveau des catégories conceptuelles, ce qui revient à dire qu'un objet peut être catégorisé avant l'acquisition du langage (ceci est un chat), mais que l'attribution à cette catégorie d'une catégorie superordonnée (un chat est un félin) présuppose la disposition du lexique approprié. La lexis est conçue comme en relation directe avec la reconnaissance phonologique mais indirecte avec la production phonologique, parce que l'enfant qui acquiert une langue adapte sa production à sa reconnaissance.

La théorie de linguistique neurocognitive de Lamb distingue deux types de relations: unimodales (catégorisation linguistique d'expression et de contenu) et multimodales (entre catégorisation linguistique et extralinguistique). À titre d'illustration (p. 152,

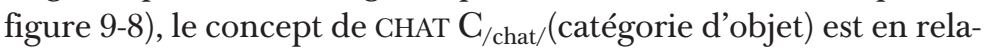
tion d'une part avec le concept de FÉLIN $\mathrm{C}_{\text {/félin/ }}$ (catégorie d'objet superordonnée) et d'autre part avec le lexème chat $\mathrm{L}_{\text {/chat }}$. Le concept de FÉLIN est en relation aussi bien avec le lexème chat qu'avec le lexème félin, ce qui fonde la proposition universelle Tout chat est un felin. Pour ce qui concerne les relations multimodales, le concept de CHAT est en relation avec le percept visuel de la silhouette d'un chat, le percept auditif de miaulement par l'intermédiaire du concept de miaulement $(\approx$ ce qui miaule, c'est les chats) et le percept tactile de la fourrure d'un chat.

Sur la base, d'une part, de ce qu'il appelle le "principe de proximité » et, d'autre part, des acquis sur le rôle du faisceau arquécomme transmetteur d'informations entre production phonologique et grammaticale (dans l'aire de Broca) et reconnaissance phonologique et grammaticale (dans l'aire de Wernicke), Lamb essaie de localiser 
grossièrement dans l'hémisphère gauche du cerveau les représentations associées au lexème chat préalablement distinguée. Sur la figure 182: Approximative locations of some nections of the concept CAT, Lamb représente par des arcs les «nexions » entre une zone affectée à la représentation lexicale et une zone affectée à la représentation conceptuelle de cat, une zone de reconnaissance et une zone de production phonologique, et des zones de reconnaissance du toucher de la fourrure du chat, de la vision d'un chat et de l'audition d'un miaulement.

Plus importante à mon sens est chez Lamb la prise en compte de la variation interindividuelle dans la corrélation entre le lexique et les concepts. Cette variation est une conséquence de deux principes fondamentaux de la linguistique neurocognitive: le système cognitif est un processeur parallèle où chaque «nexion » est son propre processeur, et l'apprentissage est un processus d'activation sélective des connexions issues de «nexions » latentes. Lamb précise que « le système linguistique couplé au système conceptuel de chaque locuteur est différent de celui de tout autre locuteur. Il n'y a donc pas de possibilité de communication parfaite par le langage » (1999: 376).

Une illustration convaincante de cette hypothèse variationniste est fournie par l'étude des « catalyses coexistantes » du lexème hamburger en anglo-américain. Un Américain moyen n'associe pas le lexème hamburger au port allemand de Hambourg. De ce fait, il n'a pas tendance à segmenter ce mot en une base lexicale hamburg-et un suffixe-er. Comme il dispose par ailleurs d'un paradigme morpholexical \{fishburger, steakburger, cheeseburger\}, il tend à segmenter hamburger en ham + burger. Pour ces mots, même si le second constituant n'a pas d'existence autonome, le premier est sémantiquement clair : il s'agit de sandwiches contenant respectivement du poisson, du steak ou du fromage. Cependant, ce n'est pas vrai du hamburger qui ne contient pas de jambon (ham) mais du steak haché. Il en résulte un conflit entre un facteur positif, la présence du paradigme morpholexical, et un facteur négatif, la disparité entre la désignation ham et le contenu du sandwich. Pour l'Américain moyen, le premier facteur l'emporte en raison de l'absence du troisième, à savoir la référence au port de 
Hambourg. En revanche, pour les Américains qui soupçonnent que la tradition de ce sandwich pourrait venir du port de Hambourg, le facteur négatif équilibre l'existence du paradigme et la segmentation en hamburg + er l'emporte.

La mise en évidence des variations interindividuelles conforte donc l'hypothèse de validité cognitive d'une conception qui se dit « relationnelle » de la corrélation entre expression et contenu linguistique par l'intermédiaire des relations multimodales. Ces variations représentent également un type de concurrence entre motivations à l'échelle de la grammaire interne d'un locuteur.

\subsection{La phylogénèse et la thèse de la co-évolution entre cerveau, esprit et langage}

\subsubsection{L'émergence de l'« instinct» du langage}

Comment ce que S. Pinker appelle «l'instinct du langage » a-t-il pu émerger à l'aube de l'espèce humaine? L'anthropologue Terrence Deacon (1997, chap. XI : «And the Word Became Flesh») défend un modèle d'évolution «baldwinien ». La théorie de Baldwin (1902) explique en quoi les comportements des individus peuvent affecter l'évolution, tout en faisant l'économie de la thèse de Lamarck selon laquelle les réponses aux exigences environnementales acquises tout au long d'une vie pourraient être transmises directement à la progéniture. Deacon en donne une illustration avec l'évolution de la tolérance au lactose chez les peuples pratiquant de longue date l'élevage: «L'emploi de lait animal comme source de nourriture, malgré des difficultés à le digérer pour certains, a favorisé la reproduction de ceux qui le toléraient » (1997 : 323).

Il s'accorde avec l'assimilation du langage à un instinct prônée par S. Pinker sur le premier volet de son argumentation, l'hypothèse primaire de l'émergence aléatoire d'un protolangage chez un ancêtre reculé de l'Homo sapiens qui aurait procuré des avantages adaptatifs conduisant à l'internalisation de certains traits cruciaux de structure linguistique. Mais il s'en écarte sur la nature de ces traits. Il argumente contre l'hypothèse secondaire de l'inscription dans le 
patrimoine génétique d'une grammaire universelle en observant que seuls les traits du langage les plus invariants et les plus généraux persistent assez longtemps pour contribuer à un effet significatif sur l'évolution à long terme du cerveau, et que les structures linguistiques ne font pas partie de ces invariants parce que le changement linguistique est infiniment plus rapide que le changement génétique. Il voit dans la référence symbolique la seule pression sélective concevable pour l'avantage massif concédé à l'apprentissage linguistique dans l'espèce humaine, considérant que « l'universalité n'est pas en elle-même un indicateur fiable de ce que l'évolution a construit dans les cerveaux humains » (ibid.: 339).

Cependant, le type d'universalité en cause est seulement celui de la Grammaire Universelle d'inspiration chomskienne, c'està-dire des universaux de catégorisation syntaxique et de règles de combinaison des catégories syntaxiques. Les universaux implicationnels de Greenberg ( $c f . \S 2.1)$ ne sont pas en cause, pas plus que les dimensions universelles de conceptualisation linguistique de Seiler (2000). Et quand Deacon conclut: « Les demandes computationnelles de symbolisation ne sont pas seulement la source majeure des pressions sélectives qui pourraient avoir produit la restructuration particulière de nos cerveaux, il est vraisemblable qu'elles sont aussi la source indirecte de toute la suite de capacités et de propensions qui constituent désormais notre "instinct" du langage » (1997: 340), on peut supposer qu'il a en tête des schèmes cognitifs universels du type proposé par Heine ( $c f . \S 2.3)$.

\subsubsection{Les deux phases successives de symbolisation lexicale et grammaticale}

T. Givón $(1995$ : 393-445; 1998) partage le point de vue de T. Deacon sur la co-évolution plausible entre le cerveau, l'esprit et le langage, mais en linguiste et typologue il précise les modalités de l'évolution de la capacité de langage en distinguant une première phase de symbolisation lexicale suivie d'une seconde phase d'émergence de la grammaire.

Givón suppose en premier lieu que le système humain de traitement du langage s'est développé primairement comme une 
extension graduelle du système de traitement de l'information visuelle. Il argumente en trois points :

(a) Dans le cerveau du primate, on a pu montrer que l'information visuelle suit deux voies à partir de l'aire primaire visuelle, la voie ventrale ou temporale pour la vision des objets et la voie dorsale ou pariétale pour l'attention visuelle et les relations spatiales (1995: 408, figure 1).

(b) La reconnaissance des objets est le précurseur visuel des concepts lexicaux et celle des relations spatiales et du mouvement est celui des informations propositionnelles sur les états et les événements (ibid.: 409).

(c) Tous les modules du cerveau concernant le langage se révèlent adjacents à un précurseur prélinguistique plausible. Or, l'aire sémantique médio-temporale qui connecte à la réception l'information conceptuelle et visuelle se situe sur la voie ventrale de traitement de l'information visuelle (ibid.: 422, figure 6).

Givón en conclut que le premier code lexical du langage humain était un code visuel et gestuel iconique, et que le passage à un code auditif-oral doit avoir eu lieu plus tard sous l'effet de différentes pressions adaptatives. Le système de codage visuel-gestuel est initialement iconique et naturel, mais non automatisé. C'est par répétition et ritualisation des actes de communication que le code de communication devient abstrait, arbitraire et automatique. À ce stade, l'iconicité originelle devient en effet une entrave. La conséquence est, selon Givón, que le système de codage gestuel perd son avantage adaptatif initial. C'est lié à l'extension des relations sociales du cercle étroit des intimes - où la communication est essentiellement manipulative, à base de commandes et de requêtes à une société ouverte sur des étrangers où des informations déclaratives explicitement codées prennent le pas.

Lavantage adaptatif des actes de langage déclaratifs est énorme, facilitant les tâches essentielles dans une société humaine plus complexe. La planification conjointe d'activités futures, la coopération et la coordination de tâches collectives, l'apprentissage à partir de l'expérience des autres et l'instruction et la transmission de valeurs culturelles et de savoir-faire techniques, tout cela est largement dépendant d'informations déclaratives (ibid.: 434). 
Givón esquisse un tableau suggestif des conditions de transition entre la phase de symbolisation lexicale et de protogrammaire et la phase ultérieure de symbolisation grammaticale. Les règles de la protogrammaire concernent l'intonation, la mise en ordre des items lexicaux, elles stipulent la disposition de l'information importante en tête, le respect de l'iconicité" chronologique, l'omission de l'information prédictible, peu importante ou non pertinente. Ces règles ne sont en fait rien d'autre que le reflet de l'organisation du traitement de l'information visuelle. Le développement de la grammaire rend possible la société complexe et diversifiée entre étrangers en permettant un discours multipropositionnel et un traitement accéléré de l'information linguistique. Cependant, la prédominance du codage grammatical n'abolit pas les principes de codage utilisés dans la protogrammaire précoce. Ils constituent en quelque sorte un noyau primaire intégré au codage grammatical abstrait et arbitraire, c'est pourquoi l'iconicité demeure un fondement sousjacent du langage en dépit de la prédominance superficielle d'un codage arbitraire.

\section{Conclusion}

Les travaux abordés dans ce chapitre attestent le rapprochement qui s'est effectué dans les deux dernières décennies du $\mathrm{XX}^{\mathrm{e}}$ siècle entre les orientations fonctionnelle et cognitive en théorie grammaticale. Ce rapprochement résulte du développement de la typologie fonctionnelle des langues qui ne tient plus seulement compte de motivations internes au langage, mais a développé à la suite de l'article pionnier de J. DuBois (1985) l'analyse du jeu complexe de compétitions et de coopérations entre motivations internes et externes (celles-ci d'ordre cognitif, énonciatif et plus particulièrement, chez W. Croft, d'ordre social) qui stabilise les langues ou les fait évoluer. Les analyses de R. Langacker sur la transitivité ou la catégorisation syntaxique vont dans le même sens que celles de Givón (1995) ou Croft (1990) parce que ces auteurs intègrent le point de vue des représentations cognitives dans leur approche 
fonctionnelle. Cette intégration permet de comprendre pourquoi les langues, tout en stabilisant des structures arbitraires, conservent un fond d'iconicité ( $c f$. Givón au § 5.2).

Il se peut que le dernier ouvrage de R. Jackendoff (2002) favorise un rapprochement plus vaste entre d'une part les « théories de la grammaire fondées sur l'usage » (le regroupement des linguistiques fonctionnelles et cognitives selon M. Tomasello) et le modèle d'« architecture parallèle ", c'est-à-dire de génération de structures de trois types, phonologiques, syntaxiques et sémantiquesconceptuelles, préconisé par Jackendoff. Trois arguments plaideraient dans ce sens:

- Jackendoff préconise un amaigrissement drastique de la composante syntaxique au profit d'un corps plus étendu de règles (morpho)phonologiques et sémantiques et de règles d'interface entre phonologie et syntaxe et entre syntaxe et sémantique. Il réfute la validité d'une grammaire centrée sur la syntaxe, ce qui va dans le même sens que, par exemple, la Functional Grammar de S. Dik $(1978,1997)$, la grammaire des rôles et de la référence de R. Van Valin ( $c f$. Van Valin et LaPollac 1997) ou la Théorie Sens-Texte de I. Mel'chuk.

- Il montre que cette architecture parallèle favorise la prise en compte à la fois des faits de langue (relevant de la grammaire interne du locuteur) et des processus du traitement en réception et en production, parce que les composantes d'interface corrèlent des structures des trois ordres de la phonologie vers la sémantique pour modéliser la réception et de la sémantique vers la phonologie pour modéliser la production.

- Il se place sur le terrain des fonctionnalistes qui, tels Givón ou Lamb, cherchent à saisir la place du langage dans le comportement cognitif global de l'individu en prenant en compte le traitement du langage au niveau neurocognitif, et de ceux qui cherchent à penser la diachronie et l'émergence des langues à partir d'un modèle biologique, tels Croft ou Deacon. Le sous-titre de son ouvrage, Brain, Meaning, Grammar, Evolution, et l'ordre de mention de ses quatre composantes sont significatifs: le développement du cerveau permet l'activité symbolique source de la signification, laquelle est stabilisée par l'émergence de la grammaire au cours de l'évolution. 
Cependant deux autres arguments vont dans le sens inverse:

- En premier lieu, Jackendoff reste fidèle à la distinction entre compétence et performance et au caractère spécifique de la faculté de langage parmi les facultés appartenant au patrimoine génétique de l'espèce humaine. Mais le point de vue du paléoanthropologue T. Deacon qui identifie l'émergence de l'espèce humaine, non à partir de critères anatomiques, mais en vertu de la seule faculté de symbolisation - il qualifie l'homme en émergence d'Homo symbolicus - et donc d'échange ritualisé de signes d'abord gestuels puis oraux, va dans le même sens, celui d'une spécificité de l'aptitude humaine au maniement sophistiqué des signes, donc d'une faculté sémiotique.

- Ensuite, Jackendoff ne s'investit pas directement dans la typologie des langues, ce qui place la controverse sur un terrain plus abstrait, en particulier à propos des universaux substantiels, formels et architecturaux, qui manquent de fondement empirique.

Une unification des trois approches parait souhaitable si l'on veut que la linguistique théorique acquière le statut d'une science à part entière. Encore faut-il s'entendre sur le modèle épistémologique auquel on la confronte. La linguistique reste encore largement tentée par le modèle des sciences physiques (ce que John Haiman a appelé physics envy). Pour les représentants de la typologie fonctionnelle des langues comme pour ceux des grammaires cognitives, « la physique est une science physique analytique, recourant largement à l'approche expérimentale, non fonctionnelle et anhistorique, tandis que la linguistique est, ou devrait être, une science sociale intégrative, employant largement l'approche comparative et observationnelle, fonctionnelle et historique » (Croft 1998 : 302). C'est sur ce terrain épistémologique que le débat entre formalistes et fonctionnalistes, esquissé au § 1 à propos de la notion fondamentale d'explication, devrait être poursuivi. 
4 - Francois.doc 25/05/04 15:00 Page 134
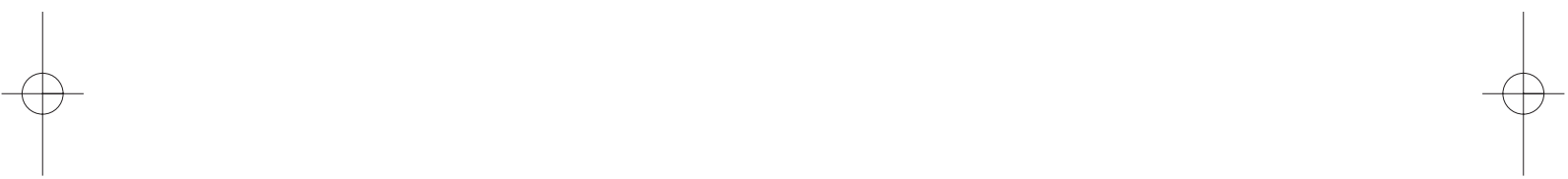